Journal of OBJECT TECHNOLOGY

Published by AITO - Association Internationale pour les Technologies Objets

http://www.jot.fm/

\title{
Introduction to the Special Issue on the Oth New Object-Oriented Languages (NOOL) Workshop in 2015
}

\author{
Alex Potanin ${ }^{\mathrm{a}}$ \\ a. Victoria University of Wellington, New Zealand
}

This JOT special issue presents 3 selected papers that formed the open call for papers following the inaugural workshop on New Object-Oriented Languages (NOOL) 2015 held in Pittsburgh, PA, USA on the 27th of October, 2015 (http: //2015.splashcon.org/track/nool2015).

The workshop idea stemmed from the fact that numerous researchers in the programming language community were involved in a large number of thoughtfully designed and implemented object-oriented languages to support their research. A lot of these languages were available for download for free hosted by repositories like GitHub and often even had small but active communities of users.

More often than not, the papers published used the language itself as a platform to demonstrate the research results and thus little definitive work addressed the actual design decisions that were made when developing a language. This workshop provided a venue for such discussion, often allowing unpublished presentation of the language design that would not prevent the authors from publishing larger research results at a later point.

The inaugural workshop was themed after the original OOPSLA 1986 Call for Papers and attracted a larger than room size number of attendees for every one of the 12 talks during the day. The annual incarnation followed with the -1st NOOL held in 2016 and -2nd NOOL expected to be held during SPLASH 2017. The reference to the $1986 \mathrm{CfP}$ was there to remind us that a lot of good ideas were around for a very long time and informed presentations connecting old ideas to new ones are very much encouraged.

Of the three papers significantly extended and presented here, the first one extends the ideas behind object-oriented multiple dispatch workshop presentation; the second combines the ideas behind the Grace inheritance mechanism as discussed during the workshop in complimentary presentations on "Classes Considered Harmful" and "Delegation is Not Quite Inheritance", while the third paper was a post-workshop contribution on the novel ideas for handling collections of data and objects.

All three papers underwent a thorough review process and underwent at least one full revision cycle before being accepted for publication in this special issue.

Alex Potanin. Introduction to the Special Issue on the Oth New Object-Oriented Languages (NOOL) Workshop in 2015. Licensed under Attribution-NoDerivatives 4.0 International (CC BY-ND 4.0). In Journal of Object Technology, vol. 16, no. 2, 2017, pages 1-2. doi:10.5381/jot.2017.16.2.e1 
About the author

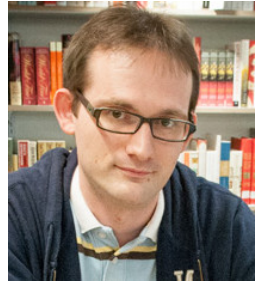

Alex Potanin is a Senior Lecturer in Software Engineering at Victoria University of Wellington, New Zealand. Having grown up from ownership, immutability, and generics, he now dabbles in secure web programming, capabilities, and effects. Contact him via email: alex@ecs.vuw.ac.nz, or visit http://homepages.ecs . vuw.ac.nz/ alex/. 